\title{
Post-seismic stress relaxation with a linear transient rheology
}

\author{
Valentina Cannelli ${ }^{1,}{ }^{\star}$, Daniele Melini ${ }^{1}$, Antonio Piersanti ${ }^{1}$ \\ ${ }^{1}$ Istituto Nazionale di Geofisica e Vulcanologia, sezione di Roma, Italy
}

\author{
Article history \\ Received March 3, 2010; accepted July 12, 2010. \\ Subject classification: \\ Transient rheology, Geodynamics, Stress tensor, Coulomb failure function, Post-seismic relaxation.
}

\section{ABSTRACT}

We performed an analysis of post-seismic stress relaxation, taking into account generalized linear rheologies. We compared the stress field (and its derived functions) obtained with a classical Maxwell rheology with that obtained with a transient Burgers body. From a set of synthetic case studies, we have revealed quantitative and qualitative differences both in relaxation times and in local stress values when a transient rheology is introduced. As a practical application, we modeled the time evolution of the Coulomb failure function following the 2009 L'Aquila earthquake, and we show that a transient rheology can lead to non-monotonic time dependence.

\section{Introduction}

Observations of post-seismic crustal deformation have often highlighted two apparently separate regimes for the relaxation behavior of the asthenosphere. Analyses focused on timescales of months to a few years have been shown to be compatible with lower crust viscosities, of $\sim 10^{17} \mathrm{~Pa} s$ [Aoudia et al. 2003, Vergnolle et al. 2003], while for longer timescales of several decades, viscosities of the order of $10^{19}$ Pa s appear to be more appropriate [Wahr and Wyss 1980, Khazaradze et al. 2002]. Most post-seismic relaxation modeling studies have adopted a Maxwell rheology to explain the viscoelastic response of the Earth at all time scales within a unified modelistic framework. As a consequence, Maxwell rheological models with asthenosphere viscosities ranging from 10 $0^{16-18} \mathrm{~Pa}$ s [Piersanti et al. 2001, Ueda et al. 2003, Hergert and Heidbach 2006] up to $10^{19-21} \mathrm{~Pa}$ s [Boschi et al. 2000, Hearn et al. 2002] have generally been reported in the literature.

This duality of behavior that in the past has represented a critical issue [Pollitz et al. 1998, Piersanti 1999, Marquart et al. 2005] can be revised in the light of the transient rheology represented by a Burgers body, as has recently been discussed in the literature [Pollitz 2003, Pollitz 2005, Hetland and Hager 2006]. Burgers rheology is a four-parameter model, the mechanical analog of which is represented by a classical Maxwell body combined in series with a Kelvin-Voigt element [e.g. Ranalli 1995]. Here, the short characteristic relaxation time of the Kelvin-Voigt element accounts for the transient state, while the longer characteristic relaxation time of the Maxwell element is associated with the steady-state.

The stress transfer due to post-seismic relaxation following strong earthquakes has been the subject of a series of studies that have endeavored to put constraints on regional viscosity [Pollitz et al. 1998] or to understand interactions among faults [Nostro et al. 2001], and the consequent geophysical implications on global seismic activity [Casarotti and Piersanti 2003]. In the present study, the role of a transient Burgers rheology in the evolution of post-seismic stress relaxation was investigated. In section 2, we recall the essentials of the background theory governing time-dependent evolution of the stress field. In section 3, we present the results obtained with a set of synthetic sources. In section 4, we demonstrate the effects of the transient rheology by simulating the time-dependent stress relaxation following the 2009 L'Aquila earthquake [Anzidei et al. 2009, Atzori et al. 2009, Walters et al. 2009].

\section{Theory}

The semi-analytical model used to compute postseismic relaxation for a spherical, incompressible, self-gravitating, layered, viscoelastic Earth has been broadly discussed in the literature, to which we refer the reader for details [Piersanti et al. 1995, Soldati et al. 1998, Boschi et al. 2000]. Here, the most important equations of the model and the mathematical formalism used for the calculation of the stress tensor are detailed for the sake of clarity.

The governing equations of the model are:

$$
\begin{gathered}
-\rho_{0} \nabla \phi_{1}-\nabla\left(u \cdot \rho_{0} g_{0} e_{r}\right)+\nabla \cdot \sigma=\rho_{0} f \\
\nabla \cdot u=0 \\
\nabla^{2} \phi_{1}=0 \\
\sigma=2 \tilde{\mu} \epsilon+p_{1} I \\
\epsilon=\frac{1}{2}\left[\nabla \cdot u+(\nabla \cdot u)^{T}\right]
\end{gathered}
$$

where $f$ is the body force equivalent to a (point-like) seismic dislocation, $\sigma$ is the incremental stress tensor, $\epsilon$ is the 
infinitesimal strain tensor, $u$ is the displacement vector, $\phi_{1}$ is the incremental potential, $g_{0}$ is the reference gravity acceleration, and $p_{1}$ is the incremental (hydrostatic) pressure. According to the correspondence principle of linear viscoelasticity [e.g. Fung 1965], all of the variables in Equations (1) implicitly depend on the Laplace variable $s$ through a "complex shear modulus» that for the Maxwell linear rheological law is:

$$
\tilde{\mu}=\frac{\mu s}{s+\mu / \eta}
$$

where $\mu$ and $\eta$ are the (elastic) shear modulus and the Newtonian viscosity for a given layer, respectively [Fung 1965]. The complex shear modulus $\tilde{\mu}_{B}$ for a Burgers body is obtained by combining in series a Maxwell and a KelvinVoigt element [Christensen 1982], such that:

$$
\tilde{\mu}_{B}=\mu_{1} s \frac{s+\mu_{2} / \eta_{2}}{\left(s+\mu_{2} / \eta_{2}\right)\left(s+\mu_{1} / \eta_{1}\right)+s \mu_{1} / \eta_{2}}
$$

where $\mu_{1}, \eta_{1}$ are the shear modulus and viscosity of the Maxwell element, respectively, and $\mu_{2}$ and $\eta_{2}$ refer similarly to the Kelvin-Voigt element.

To solve Equations (1), the displacement vector $u=\left(u_{\mathrm{r}}\right.$, $\left.u_{\theta}, u_{\phi}\right)$ is expressed in the time domain as a sum of spherical harmonics, as follows [Melini et al. 2008]:

$$
u=\sum_{l=0}^{\infty} \sum_{m=-l}^{l}\left[\begin{array}{c}
u_{l m}(r) \\
u_{l m}(r) \nabla_{\theta}+t_{l m}(r) \nabla_{\phi} \\
u_{l m}(r) \nabla_{\phi}-t_{l m}(r) \nabla_{\theta}
\end{array}\right] Y_{l}^{m}(\theta, \phi)
$$

where $\nabla=\left(\nabla_{r}, \nabla_{\theta}, \nabla_{\phi}\right)$ is the gradient operator, and $Y_{l}^{m}(\theta, \phi)$ are the spherical harmonic functions, defined as:

$$
Y_{l}^{m}(\theta, \phi)=(-1)^{m} P_{l}^{m}(\cos \theta) e^{i m \phi},
$$

with $P_{l}^{m}(z)$ being the associated Legendre functions. By substituting Equation (4) into Equations (1), the model can be linearized and the harmonic coefficients ${u_{l m}}, v_{l m}$ and $t_{l m}$ can be obtained through propagator methods [e.g. Gilbert and Backus 1966].

The stress tensor $\sigma$ is calculated using the explicit expression of $u$ (Equation 4) in Equation (1e), and then substituting the resulting deformation tensor $\epsilon$ in Equation (1d).

In the time-domain, the stress tensor $\sigma$ reads as:

$$
\begin{aligned}
& \left(\begin{array}{c}
\sigma_{r r} \\
\sigma_{r \theta} \\
\sigma_{r \phi}
\end{array}\right)=\mu \sum_{l=0}^{\infty} \sum_{m=-l}^{l}\left[\begin{array}{c}
2 \dot{u}_{l m}(r)+p_{l m}(r) \\
a_{l m}(r) \nabla_{\theta}+b_{l m}(r) \nabla_{\phi} \\
a_{l m}(r) \nabla_{\phi}-c_{l m}(r) \nabla_{\theta}
\end{array}\right] Y_{l}^{m}(\theta, \phi) \\
& \left(\begin{array}{c}
\sigma_{\theta \theta} \\
\sigma_{\theta \phi} \\
\sigma_{\phi \phi}
\end{array}\right)=\frac{2 \mu}{r} \sum_{l=0}^{\infty} \sum_{m=-l}^{l}\left[\begin{array}{c}
u_{l m}(r) \nabla_{\theta}^{2}+t_{l m}(r) \nabla_{\theta} \nabla_{\phi}+d_{l m}(r) \\
\frac{1}{2}\left(t_{l m}(r) k_{1}+u_{l m}(r)\right) k_{2} \\
u_{l m}(r) k_{3}+t_{l m}(r) k_{4}+d_{l m}(r)
\end{array}\right] Y_{l}^{m}(\theta, \phi)
\end{aligned}
$$

where the following auxiliary variables have been defined:

$$
\begin{aligned}
& a_{l m}(r)=\dot{u}_{l m}(r)-\frac{u_{l m}(r)}{r}+\frac{u_{l m}(r)}{r} \\
& b_{l m}(r)=\dot{u}_{l m}(r)-\frac{u_{l m}(r)}{r} \\
& c_{l m}(r)=\dot{t}_{l m}(r)-\frac{t_{l m}(r)}{r} \\
& d_{l m}(r)=u_{l m}(r)+p_{l m}(r) r \\
& k_{1}=\nabla_{\phi}^{2}+\tan ^{-1}(\theta) \nabla_{\theta}-\nabla_{\theta}^{2} \\
& k_{2}=\nabla_{\theta} \nabla_{\phi}-\tan ^{-1}(\theta) \nabla_{\phi}+\nabla_{\phi} \nabla_{\theta} \\
& k_{3}=\nabla_{\phi}^{2}+\tan ^{-1}(\theta) \nabla_{\theta} \\
& k_{4}=\tan ^{-1}(\theta) \nabla_{\phi}+\nabla_{\phi} \nabla_{\theta}
\end{aligned}
$$

with $p_{l m}$ being the coefficients of the incremental pressure on the spherical harmonic basis.

Once simplified by the explicit expression of the stress tensor $\sigma$ from Equation (6), the set of Equations (1) was solved analytically by Piersanti et al. [1995], with the normalmode (NM) approach originally introduced by Peltier [1974]. As widely discussed by Spada and Boschi [2006] and Melini et al. [2008], the NM technique suffers from numerical instabilities connected with the solution of the secular equation, the degree of which scales with rheological model complexity. As a consequence, layering structures with high radial resolution are practically unsolvable, and only the Maxwell rheology can be used.

Recently, a viable alternative based on the application of the «Post-Widder» (PW) formula [Post 1930, Widder 1934], was proposed in the realm of post-glacial [Spada and Boschi 2006] and post-seismic [Melini et al. 2008] deformation. The main advantage of the PW algorithm is the evaluation of the Laplace inverse of a function by sampling the values of the transform on the positive real axis, which by-passes the Residue Theorem and any root-finding procedures. On the other hand, PW code requires high floating-point precision to avoid numerical degeneration due both to its slow convergence and to the oscillating terms that can lead to catastrophic cancellation [Abate and Valk 2004, Abate and Whitt 2006].

As discussed by Spada [2008], an attractive feature of the PW formula is the straightforward implementation of general (possibly transient) linear rheological laws in addition to the Maxwell law. In the present study, for the first time, we consider the effects of the transient Burgers rheology [Yuen and Peltier 1982, Pollitz 2003] in the evaluation of postseismic stress relaxation of a spherical, self-gravitating Earth.

\section{A synthetic case study}

In what follows, we present the results obtained by applying our code to synthetic source mechanisms to evidence the transient effects on the time-dependent postseismic stress field due to a Burgers rheology. 


\begin{tabular}{|c|c|c|c|c|c|}
\hline \multirow[b]{2}{*}{ Layer $^{1}$} & \multirow{2}{*}{$\begin{array}{l}\text { Radius, } r \\
\quad(\mathrm{~km})\end{array}$} & \multirow{2}{*}{$\begin{array}{c}\text { Density, } p \\
\left(10^{3} \mathrm{~kg} \mathrm{~m}^{-3}\right)\end{array}$} & \multirow{2}{*}{$\begin{array}{l}\text { Rigidity, } \mu \\
\left(1^{10} \mathrm{~Pa}\right)\end{array}$} & \multicolumn{2}{|c|}{ Viscosity, $\eta($ Pa s) } \\
\hline & & & & Maxwell & Burgers \\
\hline \multirow[t]{5}{*}{ UC } & $6356-6371$ & 2.60 & 2.65 & $\infty$ & $\infty$ \\
\hline & $6346-6356$ & 2.90 & 4.39 & $\infty$ & $\infty$ \\
\hline & $6331-6346$ & 3.38 & 6.78 & $\infty$ & $\infty$ \\
\hline & $6311-6331$ & 3.37 & 6.76 & $\infty$ & $\infty$ \\
\hline & $6291-6311$ & 3.37 & 6.73 & $\infty$ & $\infty$ \\
\hline $\mathrm{LC}$ & $6091-6291$ & 3.40 & 6.75 & $\begin{array}{l}\eta_{1}=10^{19} \\
\eta_{2}=\infty\end{array}$ & $\begin{array}{l}\eta_{1}=10^{19} \\
\eta_{2}=5 \times 10^{17}\end{array}$ \\
\hline UM & $5701-6091$ & 3.73 & 9.40 & $10^{21}$ & $10^{21}$ \\
\hline MA & $3480-5701$ & 4.88 & 21.7 & $3 \times 10^{21}$ & $3 \times 10^{21}$ \\
\hline $\mathrm{CO}$ & $0-3480$ & 10.9 & 0 & 0 & 0 \\
\hline
\end{tabular}

Table 1. Summary of the rheologies used in the synthetic case study ( ${ }^{1}$ UC: Upper crust, LC: Lower crust, UM: Upper mantle, MA: Mantle, CO: Core).

\section{Source and rheology models}

For the synthetic study, two point-like source mechanisms were considered: a vertical, right-lateral strikeslip, and a thrust dip-slip, dipping at $20^{\circ}$. Both sources were buried at $30 \mathrm{~km}$ in depth, and their scalar moment is $M_{0}=$ $10^{21} \mathrm{Nm}$. For each source depth, we computed the stress tensor on two observation planes located at $15 \mathrm{~km}$ and 45 $\mathrm{km}$ in depth: the reason for this choice was because of the convergence of the harmonic series (Equation 6) that, in case of short source-observer radial distances, becomes slow and can lead to large round-off errors in recursive expressions of $P_{l m}$ [Riva and Vermeersen 2002, Casarotti 2003]. Details of the behavior of the solution and its convergence as a function of the harmonic degree for different source-observer distances were discussed in detail by Casarotti [2003].

In Table 1, the viscosity models used for the evaluation of the post-seismic relaxation are summarized. We considered a pure Maxwell viscoelastic model and a Burgers rheological model with a transient lower crust. The elastic structure for both of the models is characterized by a highresolution, uniformly layered upper crust and a low-resolution, uniformly layered mantle. Viscosity values for the lower crust are $\eta=10^{19}$ Pa s for the Maxwell model, and $\eta_{1}=10^{19} \mathrm{~Pa}$ s and $\eta_{2}=5 \times 10^{17} \mathrm{~Pa}$ s for the Burgers model. In the Burgers model, the transient and elastic shear moduli were assumed to be equal, i.e. $\mu_{1}=\mu_{2}$ in Equation (3). The considered viscosity profiles represent typical values on global or large scales, and were provided by post-glacial rebound data [Velicogna and Wahr 2002] and post-seismic global deformation [Ueda et al. 2003, Pollitz et al. 2006]. Regional rheological models were not considered here because of the well-known lack of sensitivity of post-seismic deformation to small-scale structures [Piersanti et al. 2001, Aoudia et al. 2003, Dalla Via et al. 2005].

\section{Post-seismic stress evolution}

Figures 1 to 4 and Supplementary Figures S1 to S4 show the time evolution of the stress field evaluated for the source mechanisms described above, computed with the two rheological models of Table 1 , on a square grid of $30^{\circ} \times 30^{\circ}$. The stress tensor components were evaluated for eight time steps ( $t=0,0.25,0.50,0.75,1,5,10,100 \mathrm{yr}$ ); to better appreciate the time evolution for $t>0$, we plotted the incremental changes in the stress tensor with respect to the previous time step, i.e. $\sigma_{i j}\left(t_{k}\right)-\sigma_{i j}\left(t_{k-1}\right)$.

For each configuration of source-observer distance, source model, and stress tensor component, differences between the two rheological models were evident only for the second $(0.25 \mathrm{yr})$ and third $(0.50 \mathrm{yr})$ temporal steps: i.e. immediately after the event. This was consistent with the mechanical behavior of a Burgers body with a low-viscosity element (see Table 1) with a (Kelvin-Voigt) characteristic time of about 0.23 yr. For longer time scales, the Maxwell and Burgers rheologies showed similar stress relaxation, since the temporal evolution of the Burgers rheology is dominated by its Maxwell element for longer time scales.

The radial components of the stress tensor appeared to be one order of magnitude less when compared with the angular components, the latter also having a relaxation process that affected a larger area, as also seen previously [Casarotti 2003]. For both source mechanisms, with the observer located at $15 \mathrm{~km}$ in depth, the radial components (Figure 2 and Supplementary Figure S2) showed degeneration problems, probably connected with incomplete convergence because of the short source- 
obs $@ 45 \mathrm{~km}$

1)
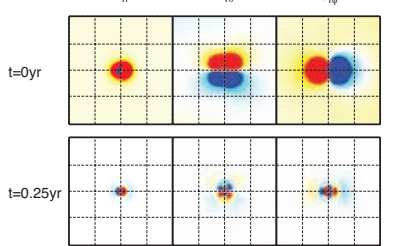

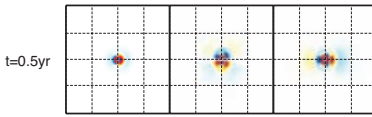
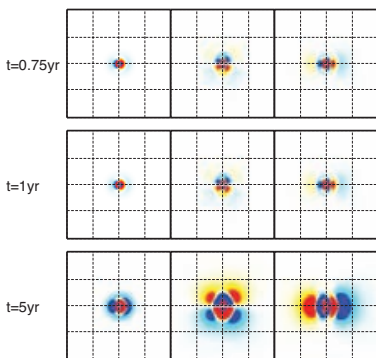

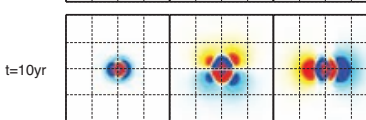
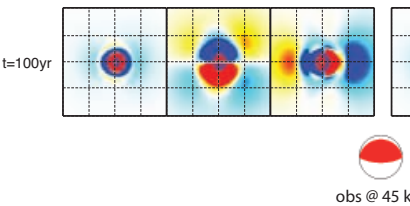

3) Max

Burg
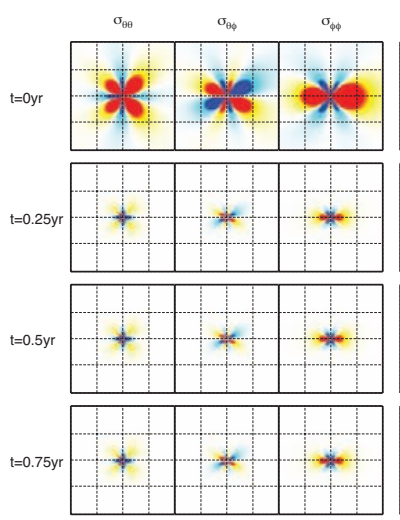

t=1yr

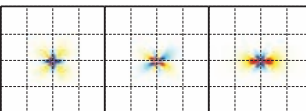

t=5yr

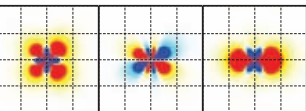

t=10yr

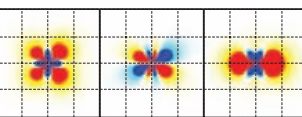

$t=100 y r$

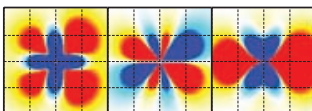

Burg
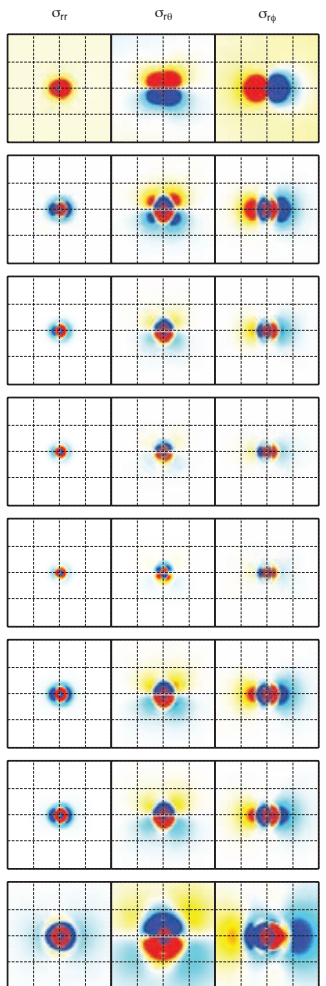

(3)
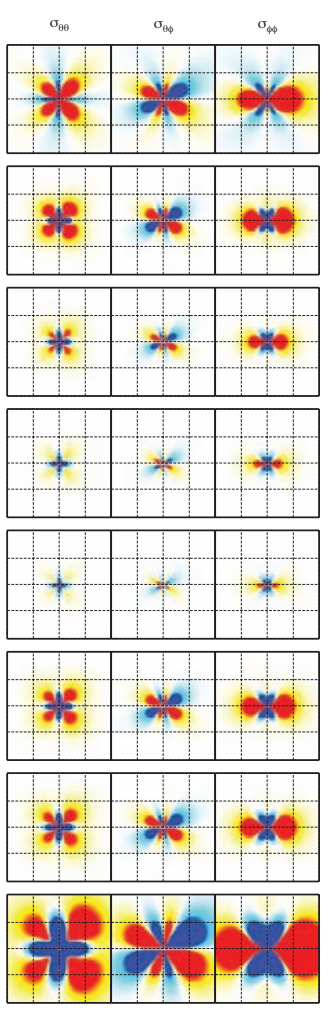
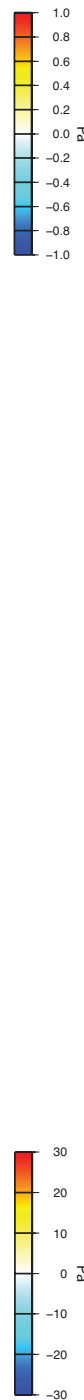

2)

obs@15 km
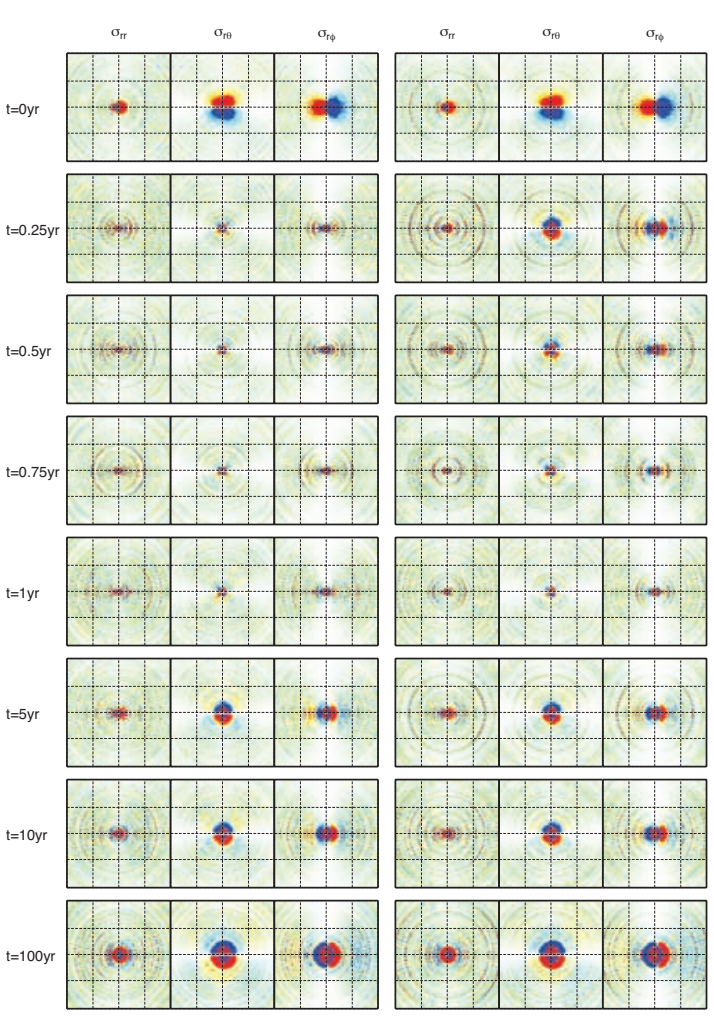

4)
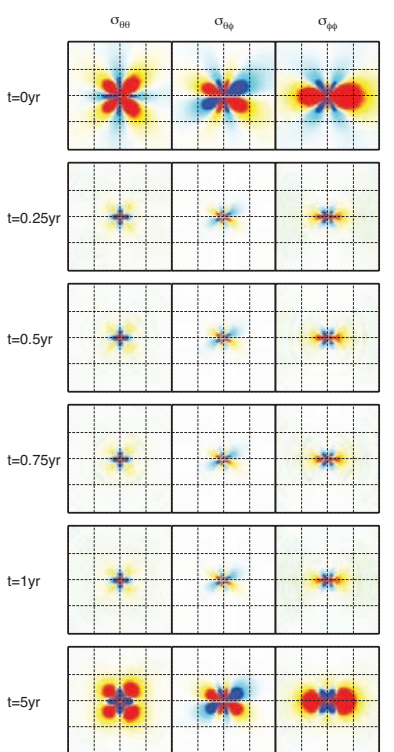

$t=10 y r$

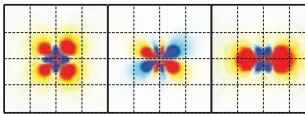

$t=100 y r$

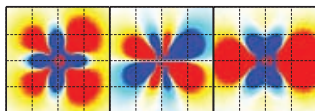

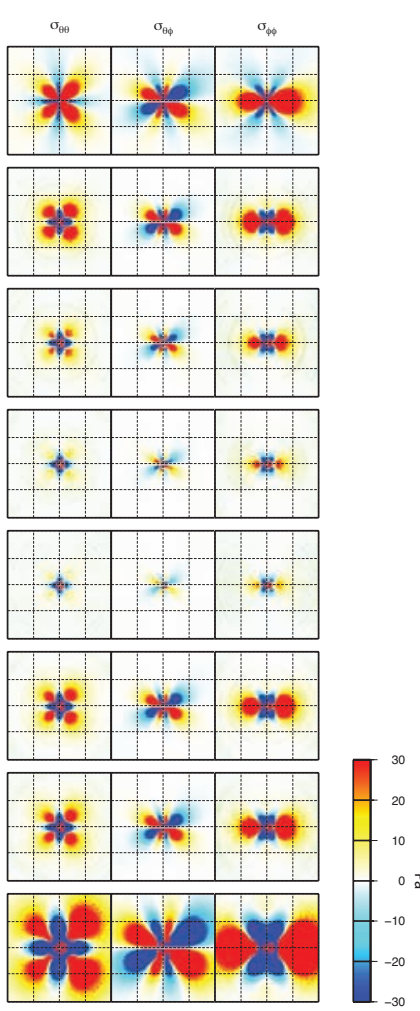

Figure 1. Temporal evolution of the radial stress tensor components $\left(\sigma_{r r}, \sigma_{r \theta}, \sigma_{r \phi}\right)$ evaluated for a point-like, dip-slip fault buried at $30 \mathrm{~km}$, according to the Maxwell (Max) and Burgers (Burg) rheologies. The scalar moment of the source is $M_{0}=10^{21} \mathrm{Nm}$, and its strike direction is towards the North. The observer is located at $45 \mathrm{~km}$ in depth. The incremental stress perturbation was evaluated at the temporal steps of $t=0,0.25,0.50,0.75,1,5,10$ and $100 \mathrm{yrs}$ (as indicated), each with respect to the previous time step, except for $t=0$, which represents the reference stress co-seismic field. The stress is expressed in Pascal (Pa). Figure 2. As for Figure 1, for an observer located at $15 \mathrm{~km}$ in depth. Figure 3. As for Figure 1, for the angular stress tensor components $\left(\sigma_{\theta \theta}, \sigma_{\theta \phi}, \sigma_{\phi \phi}\right)$ and for an observer located at $45 \mathrm{~km}$ in depth. Figure 4 . As in Figure 1 , for the angular stress tensor components $\left(\sigma_{\theta \theta}, \sigma_{\theta \phi}, \sigma_{\phi \phi}\right)$ and for an observer located at $15 \mathrm{~km}$ in depth. 


\begin{tabular}{|c|c|c|c|c|c|c|}
\hline \multirow[b]{2}{*}{ Layer $^{1}$} & \multirow{2}{*}{$\begin{array}{l}\text { Radius, } r \\
\quad(\mathbf{k m})\end{array}$} & \multirow{2}{*}{$\begin{array}{c}\text { Density, } p \\
\left(10^{3} \mathrm{~kg} \mathrm{~m}^{-3}\right)\end{array}$} & \multirow{2}{*}{$\begin{array}{c}\text { Rigidity, } \mu \\
\left(1^{10} \mathrm{~Pa}\right)\end{array}$} & \multicolumn{3}{|c|}{ Viscosity, $\eta$ (Pa s) } \\
\hline & & & & Mod2 & Mod3 & ModB \\
\hline UC & $6361-6371$ & 2.60 & 2.65 & $\infty$ & $\infty$ & $\infty$ \\
\hline $\mathrm{TZ}$ & $6351-6361$ & 2.70 & 3.25 & $10^{18}$ & $10^{18}$ & $10^{18}$ \\
\hline \multirow[t]{2}{*}{ LC } & $6336-6351$ & 2.75 & 3.37 & $10^{18}$ & $10^{17}$ & $10^{18}$ \\
\hline & & & & & & $10^{17}$ \\
\hline MA & $3480-6336$ & 4.45 & 7.35 & $10^{21}$ & $10^{21}$ & $10^{21}$ \\
\hline $\mathrm{CO}$ & $0-3480$ & 10.93 & 0 & 0 & 0 & 0 \\
\hline
\end{tabular}

Table 2. Mod2, Mod3, ModB model parameters used for the 2009 L'Aquila earthquake $\left({ }^{1}\right.$ UC: Upper crust, TZ: Transition zone, LC: Lower crust, MA: Mantle, CO: Core).

observer distance [Casarotti 2003]. The small amplitude of radial components was also likely to have had a role, due to the larger relative numerical errors [Casarotti 2003].

It is interesting to observe that the presence of a transient layer implies not only a shorter time scale for the relaxation process, but it also had a sensitive impact on the overall different shape of the stress field: in Figure 1 and Supplementary Figure S1 (radial components), using the Maxwell and Burgers rheologies, the patterns corresponding to the second time step $(0.25 \mathrm{yr})$ had a different distribution of lobes. The same was observed for the angular components in Figures 3 and 4 for the second and third time steps, i.e. 3 and 6 months after the event, respectively.

The former represents the general behavior, except for the angular components corresponding to the configuration with the strike-slip fault and the observation planes located at $45 \mathrm{~km}$ and $15 \mathrm{~km}$ in depth (Supplementary Figures S3 and S4, respectively), for which the two rheologies showed only different relaxation velocities, corresponding to different amplitudes of the lobes.

This synthetic case study revealed that a transient rheology accelerates the initial phase of the relaxation process, which is a well-known phenomenon that has been thoroughly investigated in the literature [Pollitz 2003, Pollitz 2005, Hetland and Hager 2006]. Furthermore, a transient rheology causes an overall distortion of the relaxation pattern, giving rise to local stress values that are different from those obtained with a Maxwell rheology, thus eventually implying different physical consequences, e.g. when stress relaxation is used to assess fault-interaction effects.

\section{Stress relaxation following the 2009 L'Aquila earthquake}

In this section, as a practical application, we discuss the post-seismic stress relaxation following the April 6, 2009, L'Aquila earthquake [Anzidei et al. 2009, Atzori et al. 2009, Walters et al. 2009]. We modeled the seismic source as a 1-D fault segment with a length of $13.0 \mathrm{~km}$, a dip angle of $55.3^{\circ}$, and a cumulative seismic moment $M_{0}=3.2 \times 10^{18} \mathrm{Nm}$, obtained as a linear superimposition of 30 equally spaced, point-like dislocations. This approximation procedure for the seismic source has been shown to provide reliable results also in the near field [Piersanti et al. 1997]. The center of the fault was located at $42.32^{\circ} \mathrm{N}, 13.42^{\circ} \mathrm{E}$, at a depth of $9.5 \mathrm{~km}$. The seismic source parameters used were those resulting from the inversion described by Anzidei et al. [2009], except for the depth, which was fixed according to INGV [2009]. The observation planes were located at $\pm 5 \mathrm{~km}$ from the source center, to ensure the convergence of the solution, as discussed in Section 3.

In Table 2, the three stratification models chosen for the evaluation of this post-seismic relaxation are given. The models labeled as Mod2 and Mod3 were the best-fitting models obtained by Aoudia et al. [2003], from the postseismic relaxation following the 1997 Umbria-Marche (central Apennines) earthquake. ModB, which combined the Maxwell and Burgers rheologies, represents a possible synthesis [Pollitz 2003] of the two models obtained by Aoudia et al. [2003] through considering only the Maxwell rheologies. The aim was to take into account both short relaxation times, consistent with the presence of a lowviscosity Kelvin-Voigt element, and longer time scales, connected with a Maxwell element.

\section{Stress tensor evolution}

Figures 5 to 10 show the time evolution of the stress tensor, evaluated for the three rheological stratifications summarized in Table 2, on a grid with an extension from $12.5^{\circ}$ to $14.1^{\circ}$ in longitude, and from $41.5^{\circ}$ to $43.1^{\circ}$ in latitude. The temporal steps were $t=0,1,2,3,6$ and 12 months. The $t=0$ panels show the reference co-seismic stress field, while for $t>0$, we plotted the incremental stress changes with respect to the previous time step.

Some comments that are valid for the synthetic case are confirmed by this practical application: for all of the considered rheologies and for each observation depth, the amplitudes of the radial components were clearly smaller 
5)

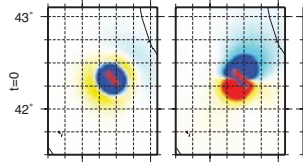

${ }_{42}^{42^{3}}$

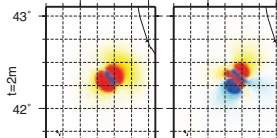

$+1+-1$

${ }_{42}^{52}$

$++++$

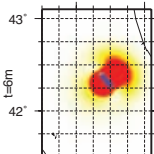

${ }^{43^{\circ}}+$

$2+1,1,1$ obs@14.46 km

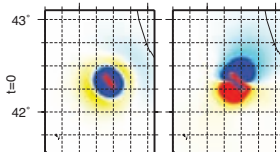

Iㄴ.

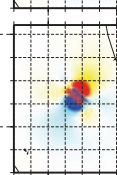

${ }_{42^{2}}{ }^{43^{\circ}}$

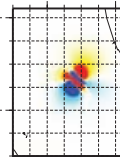

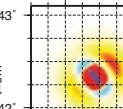

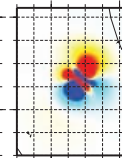

(3)

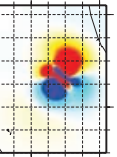

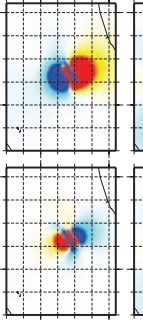
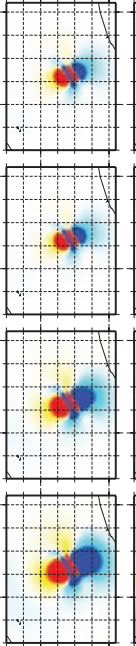

obs@14.46 km
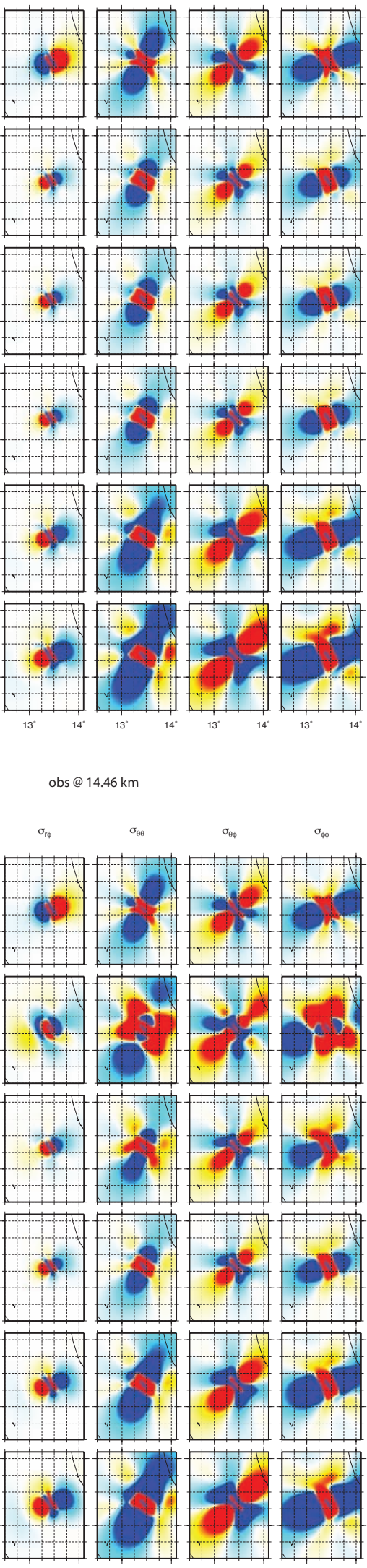

6)
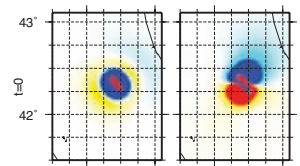

1

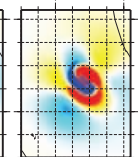

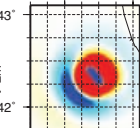
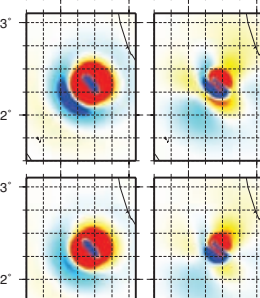

$+1-1$
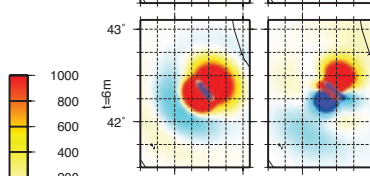

$0_{\Xi^{4}}^{43^{\circ}}=-+\cdots$

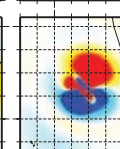

$2+\cdots$

obs@ $14.46 \mathrm{~km}$

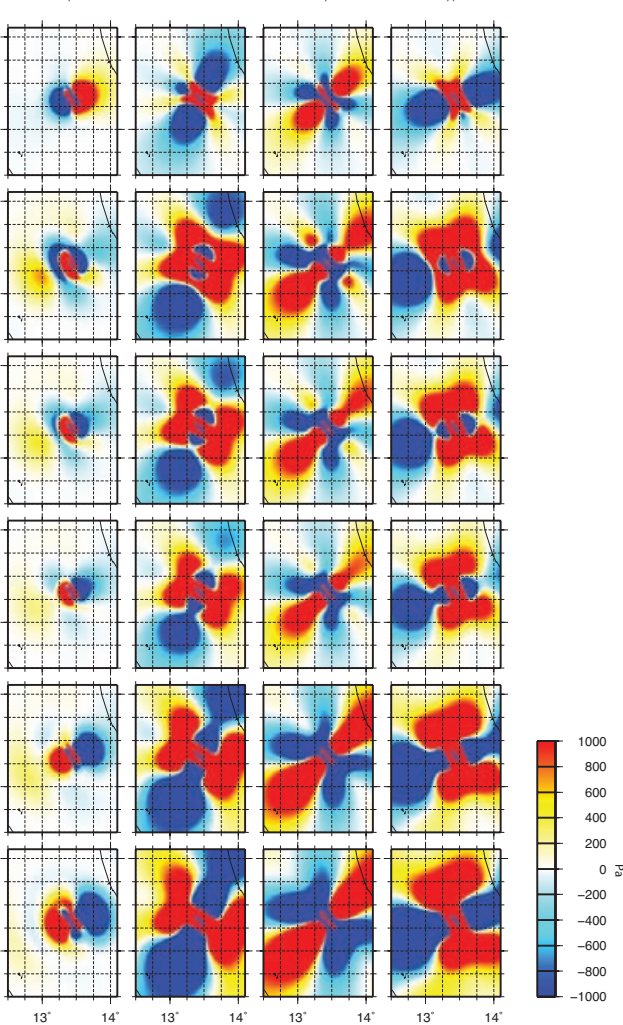

obs @ $4.46 \mathrm{~km}$
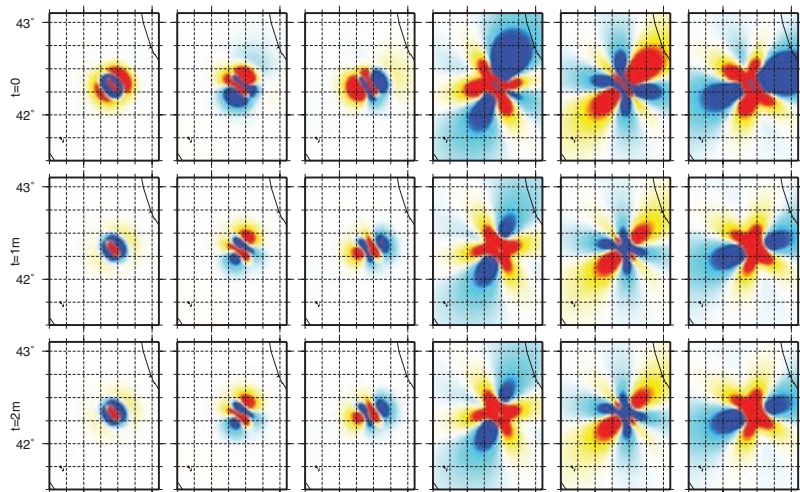

Iㄴ.
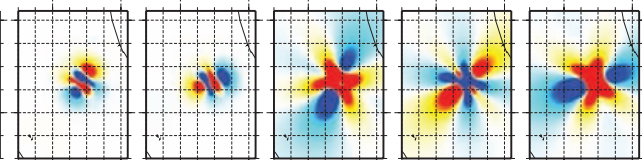

${ }_{42}^{42^{2}}$
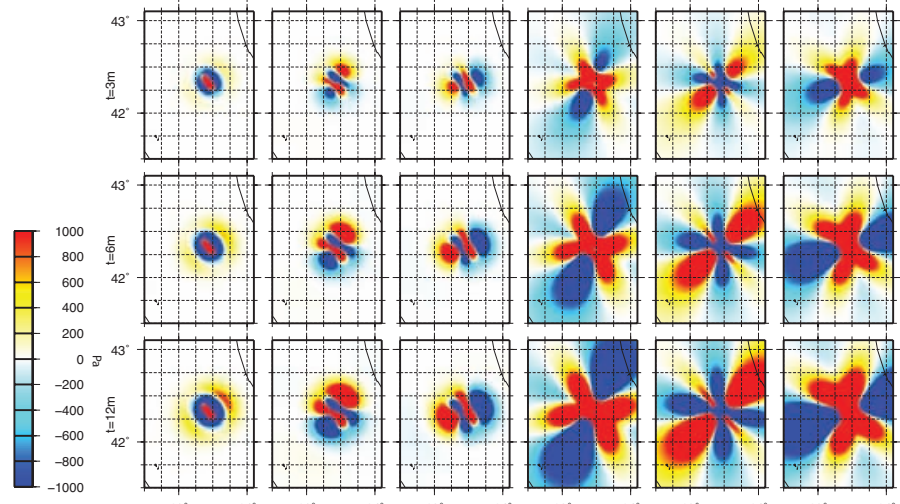

$\left[\begin{array}{r}1000 \\ 800 \\ 600 \\ 400 \\ 200 \\ 0 \\ -200 \\ -400 \\ -600 \\ -800 \\ -1000\end{array}\right.$

Figure 5. Temporal evolution of the stress tensor evaluated for the 2009 L'Aquila earthquake, with rheological model Mod2 (see Table 2) for the temporal steps of $t=0,1,2,3,6$ and 12 months (as indicated), each with respect to the previous time step, except for $t=0$, which represents the reference stress co-seismic field. The observer is located at $14.46 \mathrm{~km}$ in depth. The stress is expressed in Pascal (Pa). For the first step, the color scale range has to be multiplied by a factor of 10 . Figure 6. As for Figure 5, with rheological model Mod3 (see Table 2), and the observer located at $14.46 \mathrm{~km}$ in depth. Figure 7 . As for Figure 5, with rheological model ModB (see Table 2), and the observer located at $14.46 \mathrm{~km}$ in depth. Figure 8. As for Figure 5, with rheological model Mod2 (see Table 2), and the observer located at $4.46 \mathrm{~km}$ in depth. 
9)

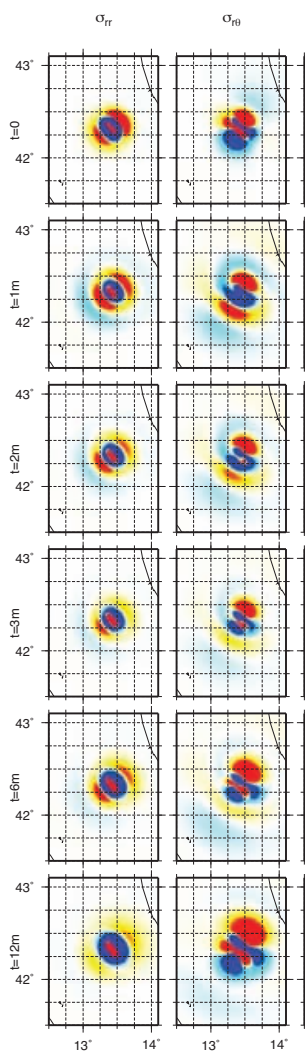

obs@ $4.46 \mathrm{~km}$
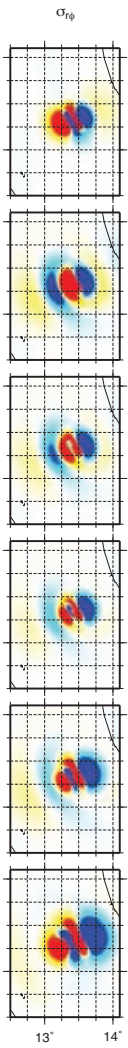
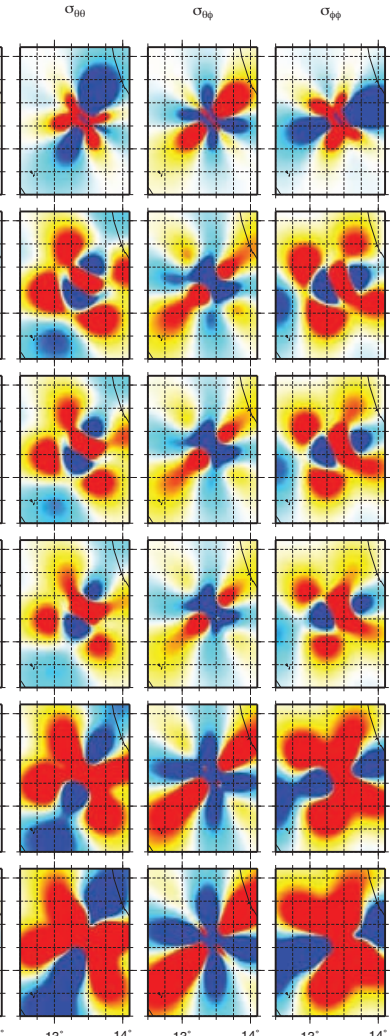

10)
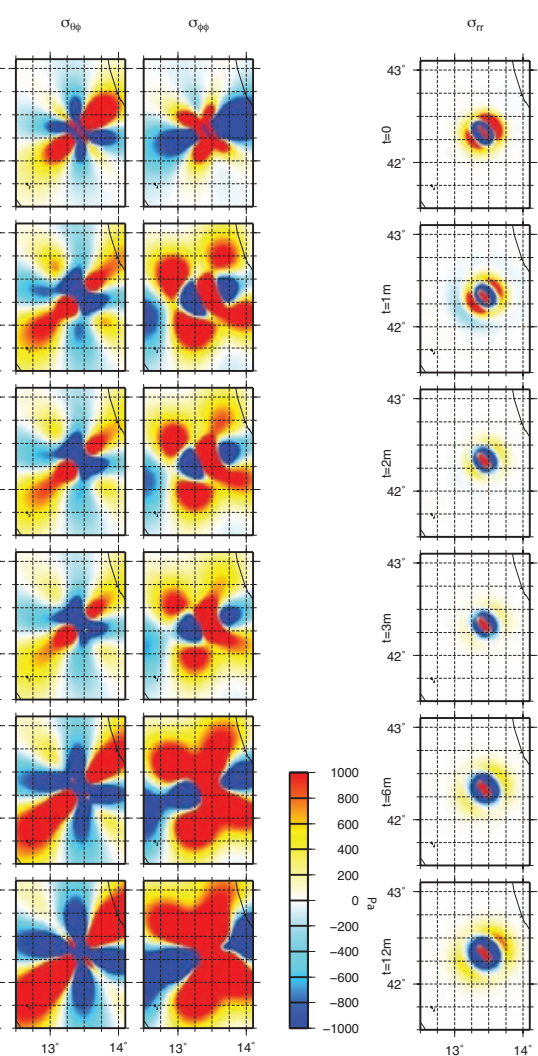
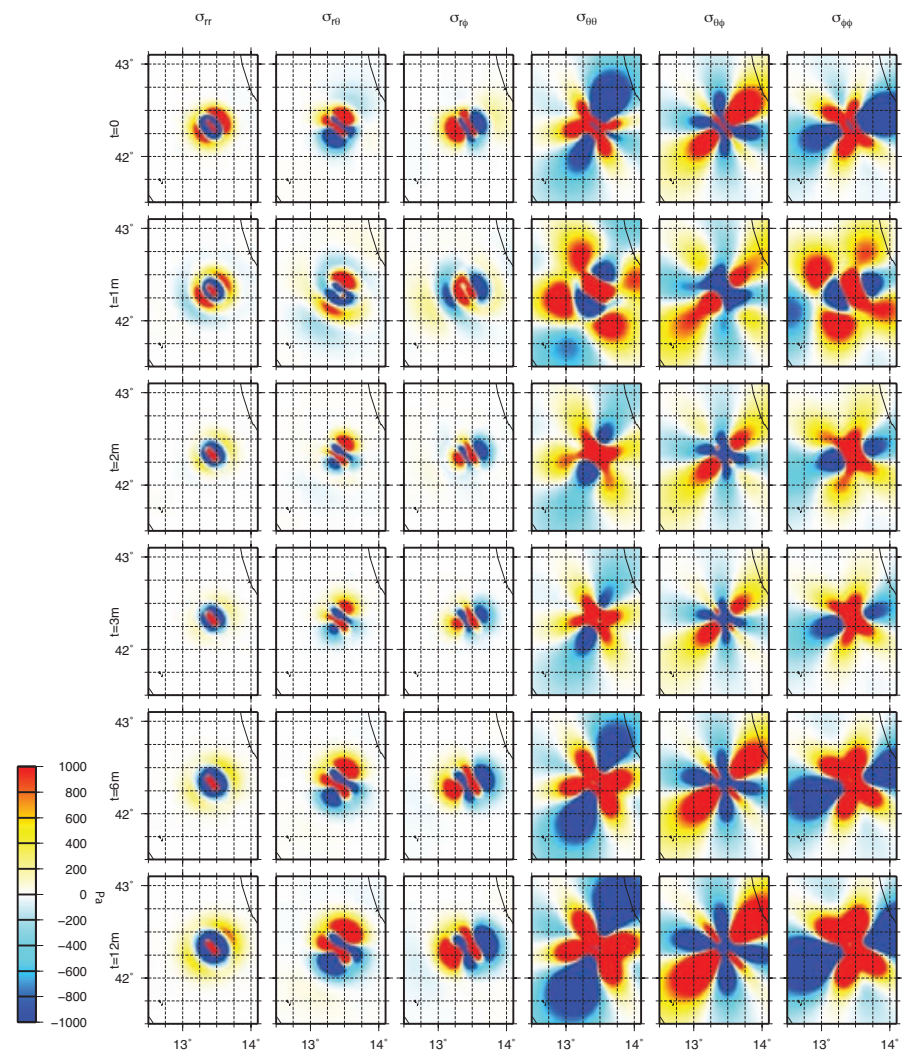

Figure 9. As for Figure 5, with rheological model Mod3 (see Table 2), and the observer located at $4.46 \mathrm{~km}$ in depth. Figure 10. As for Figure 5, with rheological model ModB (see Table 2), and the observer located at $4.46 \mathrm{~km}$ in depth.

than those of the angular ones. However, Figures 5 to 10 show a large area loaded by stress variation from the fourth time step on. Clearly, because of the distinct relaxation times of the Mod2 and Mod3 models ( $\sim 11$ months, and $\sim 1$ month, respectively), the evolution of the stress tensor for these two rheological models was quite different in the explored time window (while not particularly interesting for the present study).

The behavior of the ModB rheological model is instead worth noting: at the second time step (one month after the event), the results for the ModB and Mod3 models are similar, with Mod3 showing the same relaxation time of the transient element of ModB. Small differences are found, however, in the angular components in terms of lobe extensions and both positive and negative stress concentrations (see Figures 6, 7 and 9, 10). The choice of a scale range suitable for all of the stress tensor components and for all of the time steps presented here penalizes the display of such similar differences existing also for the radial components. From the third time step on, the ModB model began to converge towards the Mod2 model, which represents the limit of the ModB model for $t \rightarrow \infty$, showing small differences only in terms of the lobe extension, even if it is less pronounced. Thus, from a qualitative point of view, we can indicate that the inclusion of a transient rheology represents in this case a possible modelistic synthesis for different relaxation regimes, which allows the reconciliation of the two Maxwell viscosities obtained by Aoudia et al. [2003] in an analysis of short-term and long-term relaxation.

\section{Coulomb failure function variation}

To gain better insight into the impact of transient rheology in the post-seismic stress relaxation process, as a further example, we computed the effects of different linear viscoelastic rheologies on the Colulomb failure function (CFF) associated with the 2009 L'Aquila event. The $\Delta \mathrm{CFF}$ variation is defined as $\Delta \mathrm{CFF}=\Delta \tau+\mu \Delta \sigma$, where $\Delta \tau$ is the change in shear stress evaluated along the slip direction on the assumed fault plane, $\Delta \sigma$ is the change in normal stress (positive for extension), and $\mu$ is the apparent friction coefficient after accounting for the pore fluid pressure effects [King et al. 1994]. We obtained the postseismic CFF variation by projecting the six components of the stress tensor, which were previously evaluated for observation planes located at $\pm 5 \mathrm{~km}$ from the source, on the fault plane defined by the focal mechanism solution. The apparent friction coefficient was set to 0.3 , according to Catalli et al. [2008].

Figures 11 and 12 show the temporal evolution of the CFF, as evaluated for the three rheological stratifications summarized in Table 2 on the same grid used for the stress relaxation, for temporal steps $t_{i}=0,10,20,30,60,90,120$ and 180 days. For each temporal step, $t_{i}$, we also reported the distribution of the aftershocks that occurred at $t_{i} \pm \Delta t$ (as 
11)
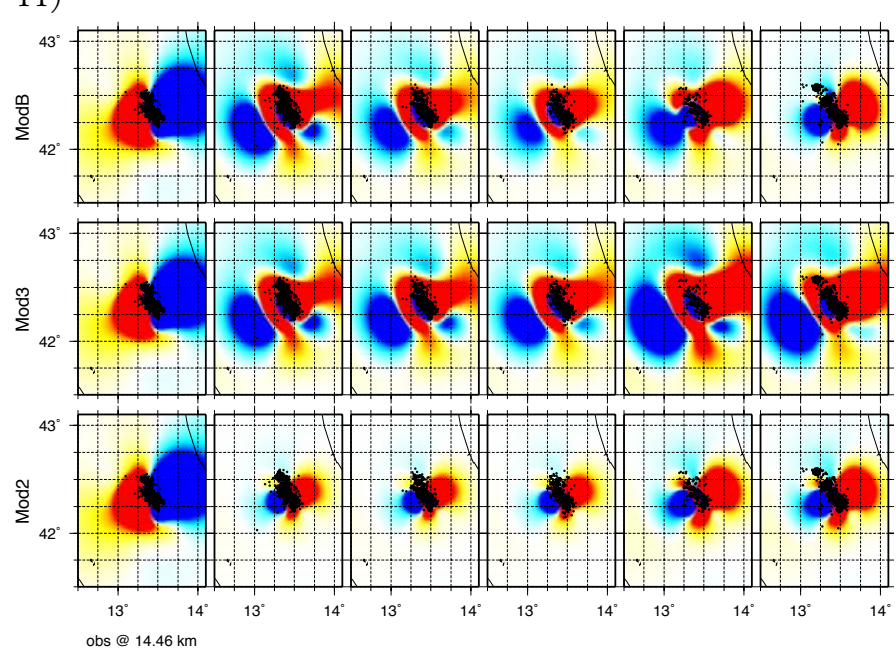

12) $\quad t=0 \quad t=10 d \quad t=20 d \quad t=30 d \quad t=60 d \quad t$
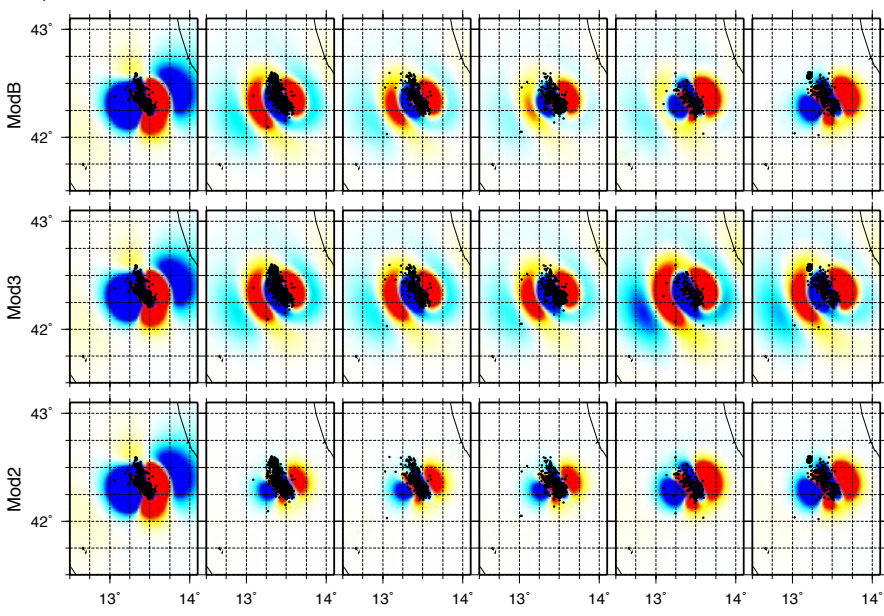

Figure 11. Temporal evolution of the post-seismic CFF associated with the 2009 L'Aquila earthquake, for the different rhelogical models (as indicated; see Table 2) at the temporal steps of $t=0,10,20,30,60$ days, and 3, 4 and 6 months (as indicated), each with respect to the previous time step, except for $t=0$, which represents the reference co-seismic CFF. The observer is located at $14.46 \mathrm{~km}$ in depth. The CFF is expressed in Pascal (Pa). For the first step, the color scale range has to be multiplied by a factor of 10 . For each temporal step, the distribution of aftershocks with depth $\geq 9.46 \mathrm{~km}$ is also shown (black dots) Figure 12. As for Figure 11, with the observer located at $4.46 \mathrm{~km}$ of depth. For the first step, the color scale range has to be multiplied by a factor of 5 . For each temporal step, the distribution of aftershocks with depth $\leq 9.46 \mathrm{~km}$ is also shown (black dots).

$\Delta t=5$ days for $t_{i} \leq 60$ days, and $\Delta t=15$ days for $t_{i} \geq 90$ days), with depths $\geq 9.46 \mathrm{~km}$ for the observer located at $5 \mathrm{~km}$ below the source and with depths $\leq 9.46 \mathrm{~km}$ for the observer located at $5 \mathrm{~km}$ above the source.

The analysis of the CFF temporal evolution revealed that the results obtained with ModB were very similar to those with Mod3 for the first temporal steps (until about two months after the event), and to those with Mod2 for the subsequent steps, regardless of the observation plane. As already seen for the synthetic case, model ModB shows a behavior that is in agreement with the presence of a lowviscosity element $\left(10^{17} \mathrm{~Pa}\right.$ s for Mod3) immediately after the event (transient state), while for the longer time scales (steady-state), the response was comparable to that of a Maxwell rheology ( $10^{18} \mathrm{~Pa}$ s for Mod2), consistent with the expected dynamics of the mechanical analog of a Burgers body. Besides this, most of the aftershocks appeared to be located where the variation in CFF on the fault plane was positive, consistent with the hypothesis that transient stress had a role in the evolution of the sequence, even if from this simple application we cannot determine the preferred rheological model (which would anyway be beyond the aim of the present study).

To better appreciate the effects of the transient rheology on the post-seismic relaxation, we evaluated the CFF time histories at two distinct observation points, selected on the two opposite sides with respect to the fault strike (Figure 13, $P_{1}$; Figure 14, $P_{2}$; and black lines in their insets). In Figures 13 and 14 we show the results for $P_{1}$ and $P_{2}$ for the observers located at $\pm 5 \mathrm{~km}$ in depth from the source. The different curves represent the CFF time histories for the rheological stratifications summarized in Table 2. A time window of two months was sufficient to appreciate the different behavior of the three models: regardless of trend (increasing or decreasing) and sign (positive or negative), the CFF time histories showed a nearly linear trend for Mod2 and Mod3, as expected on this time scale, being relaxation times $\sim 11$ months and $\sim 1$ month, respectively. In contrast, from early time steps, ModB produced a more complex and potentially more realistic rate of change. In Figure 13a, a slope change in the CFF evaluated in $P_{1}$ is clearly evident for the observer above the source: the time history has a monotonic growth until 50 days after the event, beyond which it began to decrease slightly. The same general trend was found for $P_{2}$ for the observer below the source (Figure 14b); here, the change in the slope occurred one month after the event. It is of note that this behavior is in agreement with the results of Hetland and Hager [2006], who obtained non-monotonic post-seismic relaxation only when a transient layer with Burgers rheology was included in the layering model.

\section{Conclusions}

For the first time, we have modeled the post-seismic stress field adopting a rheological model with a transient lower crust within a spherical, self-gravitating Earth model. This approach was possible due to the PW Laplace inversion algorithm that allowed investigation of layering structures with arbitrary resolution and generalized linear rheologies within the NM framework.

The results obtained for the synthetic case study showed that a Burgers rheology for the lower crust with a steadystate viscosity of $\eta_{1}=10^{19} \mathrm{~Pa}$ s and a transient viscosity of $\eta_{2}=5 \times 10^{17} \mathrm{~Pa}$ s gives rise to a rapid transient over the first 3 months, followed by slower relaxation over the following century. This lower-crust rheology finds its motivation in the results from several studies that obtained at least two 
13a)

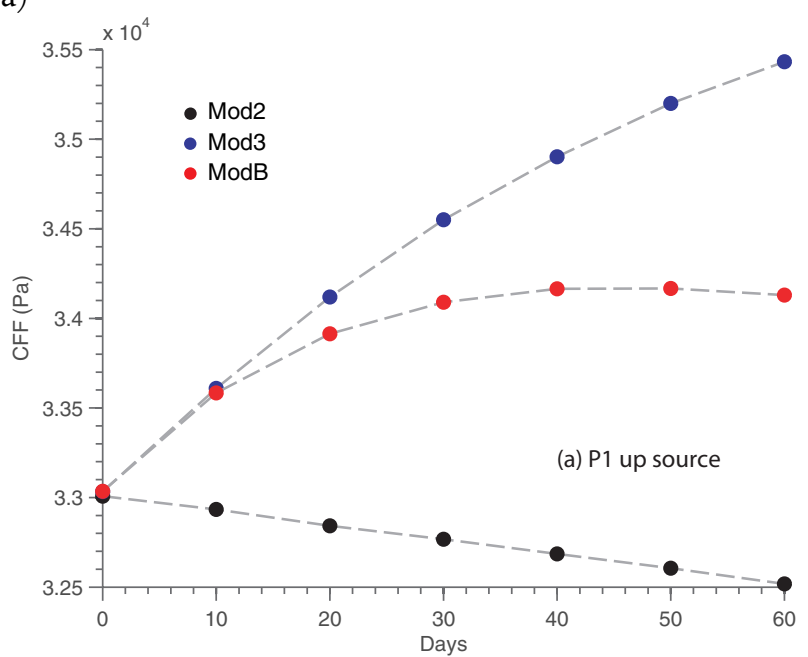

$13 \mathrm{~b})$

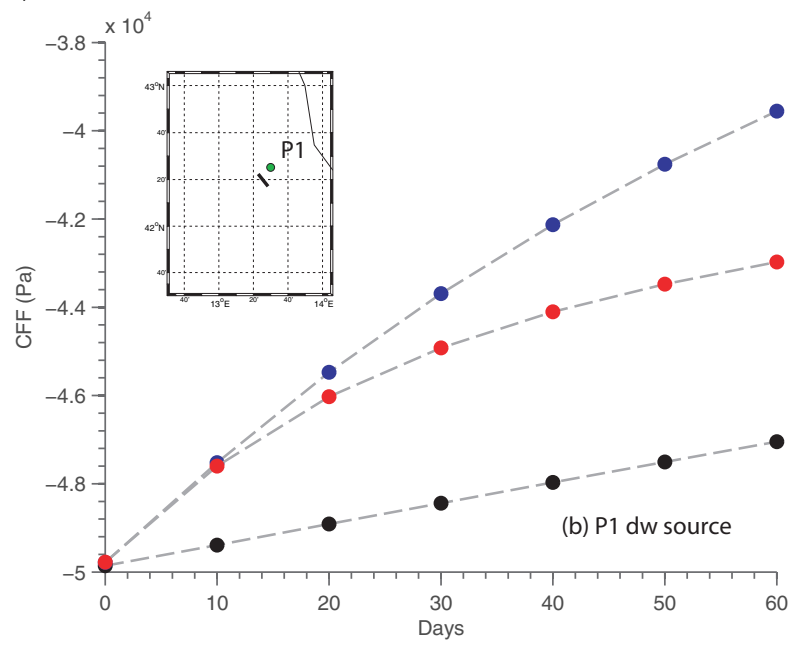

$14 \mathrm{a})$

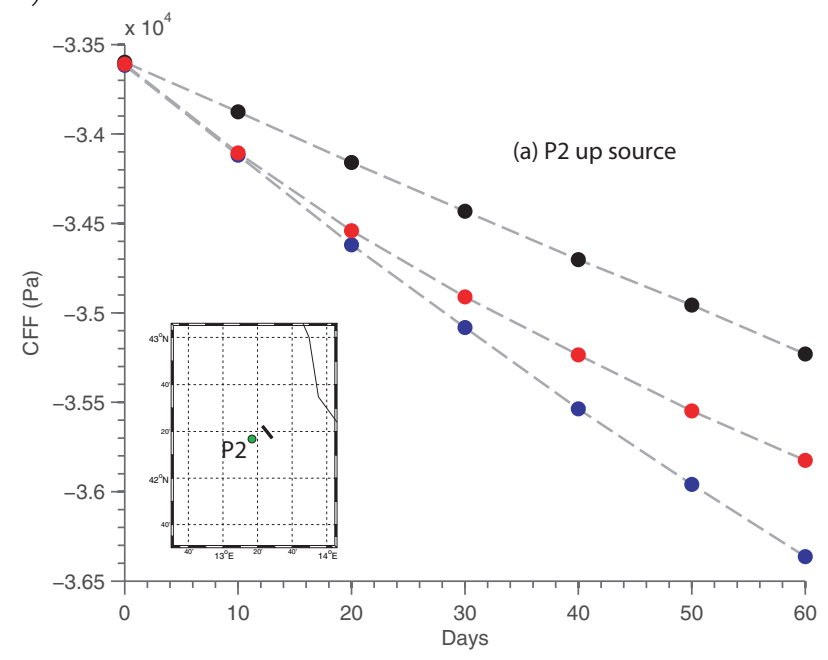

14b)

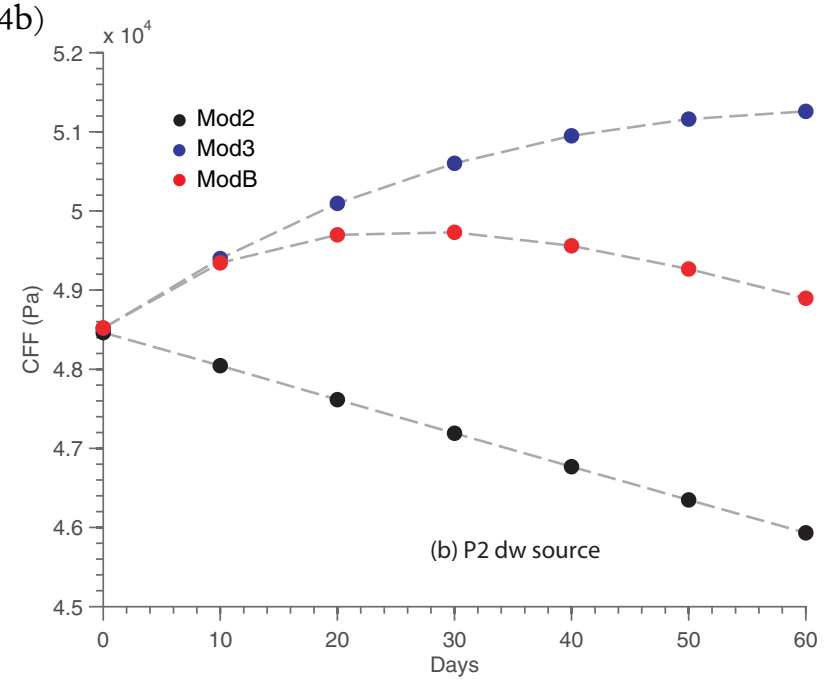

Figure 13. Time histories for the CFF associated with the 2009 L'Aquila earthquake, evaluated at location P1 (green circle in inset), for the different rheological models (as indicated; see Table 2). The observer is located at a depth of $4.46 \mathrm{~km}$ (a) and $14.46 \mathrm{~km}$ (b). The black line in the inset marks the surface projection of the modeled source. Figure 14. As for Figure 13, evaluated at location P2.

different Maxwell viscosities, depending on the datasets used [Piersanti 1999, Pollitz 2003, Pollitz 2005]. Moreover, a comparison between these Maxwell and Burgers rheologies revealed opposite stress concentrations: this might have an important role in the investigation of fault interactions through stress tranfer [Stein 1999, Casarotti and Piersanti 2003, Lin and Stein 2004], as well as in physical models of fluid migration, which are known to have a role in the seismogenic process [Miller et al. 2004, Lucente et al. 2010].

The practical application with the post-seismic stress relaxation that followed the 2009 L'Aquila earthquake, and particularly the analysis of the associated CFF variations, appear to strengthen the hypothesis that a transient rheology can account for a more realistic time dependence. The associated CFF time histories on a couple of representative points showed non-monotonic temporal dependence when a Burgers model was used; a similar feature has already been observed by Hetland and Hager [2006] under different conditions. Such temporal evolution can represent a more realistic scenario than a simple Maxwell approximation, which cannot explain the complex post-seismic relaxation observed by preliminary strain measurements [Amoruso and Crescentini 2009], regardless of the precise deformative mechanism to which it can be associated. The large amount of post-seismic data that will be available due to the deployment of a dense geodetic network in the L'Aquila region will allow more precise assessments of the rheological properties of the studied area.

Acknowledgements. We thank Giampiero Iaffaldano and an anonymous reviewer for their helpful and incisive comments. This work was partly supported by MIUR (Ministero dell'Istruzione, dell'Università e della Ricerca) with the FIRB grant «Sviluppo di nuove tecnologie per la protezione e la difesa del territorio dai rischi naturali». The Figures were prepared with the GMT software by Wessel and Smith [1991]. The source code used for the numerical simulations is available by email request to Valentina Cannelli.

\section{References}

Abate, J. and P.P. Valkó (2004). Multi-precision Laplace transform inversion, Intl. J. Numeric. Meth., 60, 979-993.

Abate, J. and W. Whitt (2006). A unified framework for 
numerically inverting Laplace transforms, INFORMS J. Comp., 18, 408-421.

Amoruso, A. and L. Crescentini (2009). Slow diffusive fault slip propagation following the 6 April 2009 L'Aquila earthquake, Italy. Geophys. Res. Lett., 36, L24306.

Anzidei, M., E. Boschi, V. Cannelli, R. Devoti, A. Esposito, A. Galvani, D. Melini, G. Pietrantonio, F. Riguzzi, V. Sepe and E. Serpelloni (2009). Co-seismic deformation of the destructive April 6, 2009 L'Aquila earthquake (central Italy) from GPS data, Geophys. Res. Lett., 36, L17307.

Aoudia, A., A. Borghi, R. Riva, R. Barzaghi, B.A.C. Ambrosius, R. Sabadini, L.L.A. Vermeersen and G.F. Panza (2003). Post-seismic deformation following the 1997 UmbriaMarche (Italy) moderate normal faulting earthquakes, Geophys. Res. Lett., 30, 1390.

Atzori, S., I. Hunstad, M. Chini, S. Salvi, C. Tolomei, C. Bignami, S. Stramondo, E. Trasatti, A. Antonioli and E. Boschi (2009). Finite fault inversion of DInSAR coseismic displacement of the 2009 L'Aquila earthquake (central Italy), Geophys. Res. Lett., 36, L15305.

Boschi, L., A. Piersanti and G. Spada (2000). Global postseismic deformation: Deep earthquakes. J. Geophys. Res., 105 (B1), 631-652.

Casarotti, E. (2003). Postseismic stress diffusion in a spherical viscoelastic Earth model, $\mathrm{PhD}$ thesis, Università degli Studi di Bologna, Italy.

Casarotti, E. and A. Piersanti (2003). Postseismic stress diffusion in Chile and South Peru, Earth and Planetary Science Letters, 206 (3-4), 325-333.

Catalli, F., M. Cocco, R. Console and L. Chiaraluce (2008). Modeling seismicity rate changes during the 1997 UmbriaMarche sequence (central Italy) through a rate-and state-dependent model, J. Geophys. Res., 113, B11301.

Christensen, R.M. (1982). Theory of Viscoelasticity, Academic Press, New York.

Dalla Via, G., R. Sabadini, G. De Natale and F. Pingue (2005). Lithospheric rheology in southern Italy inferred from postseismic viscoelastic relaxation following the 1980 Irpinia earthquake, J. Geophys. Res., 110, B06311.

Fung, Y.C. (1965). Foundations of solid mechanics, Upper Saddle River, New Jersey, USA.

Gilbert, F. and G.E. Backus (1966). Propagator matrices in elastic wave and vibration problems, Geophysics, 31 (2), 326-332.

Hearn, E.H., B.H. Hager and R.E. Reilinger (2002). Viscoelastic deformation from North Anatolian Fault Zone earthquakes and the eastern Mediterranean GPS velocity field, Geophys. Res. Lett, 29 (11), 1549.

Hergert, T. and O. Heidbach (2006). New insights into the mechanism of postseismic stress relaxation exemplified by the 23 June $2001 \mathrm{Mw}=8.4$ earthquake in southern Peru, Geophys. Res. Lett, 33, L02307.

Hetland, E.A. and B.H. Hager (2006). The effects of rheological layering on post-seismic deformation, Geophysical Journal International, 166 (1), 277-29.

INGV (2009). Istituto Nazionale di Geofisica e Vulcanologia (INGV): The L'Aquila seismic sequence - April 2009, http:/ / portale.ingvit/primo-piano-1/ news-archive/2009news/april-6-earthquake/ copythe-1-aquila-seismic-sequence -april-2009/view?set_language $=$ en (accessed on January 2010).

Khazaradze, G., K. Wang, J. Klotz, Y. Hu and J. He (2002). Prolonged postseismic deformation of the 1960 great Chile earthquake and implications for mantle rheology, Geophys. Res. Lett., 29 (22), 2050.

King, G.C.P, R.S. Stein and J. Lin (1994). Static stress changes and the triggering of earthquakes, Bull. Seismol. Soc. Am., 84, 935-935.

Lin, J. and R.S. Stein (2004). Stress triggering in thrust and subduction earthquakes and stress interaction between the southern San Andreas and nearby thrust and strikeslip faults, J. Geophys. Res., 109, B02303.

Lucente, F., P. De Gori, L. Margheriti, D. Piccinini, M. Di Bona, C. Chiarabba and N. Piana Agostinetti (2010). Inside the preparatory phase of a destructive earthquake: precursory phenomena of the April 6th 2009 L'Aquila shock in Italy, submitted on Nature Geosci., 2010.

Marquart, G., B. Steinberger and K. Niehuus (2005). On the effect of a low viscosity asthenosphere on the temporal change of the geoid - A challenge for future gravity missions, Journal of Geodynamics, 39 (5), 493-511.

Melini, D., V. Cannelli, A. Piersanti and G. Spada (2008). Postseismic rebound of a spherical earth: new insights from the application of the post-widder inversion formula, Geophysical Journal International, 174 (2), 672-695.

Miller, S.A., C. Collettini, L. Chiaraluce, M. Cocco, M. Barchi and B.J.P. Kaus (2004). Aftershocks driven by a highpressure CO2 source at depth, Nature, 427, 724-727.

Nostro, C., A. Piersanti and M. Cocco (2001). Normal fault interaction caused by coseismic and postseismic stress changes, J. Geophys. Res., 106 (B9), 19,391-19,410.

Peltier, W.R (1974). The impulse response of a Maxwell Earth, Rev. Geophys. Space Phys., 12, 649-669.

Piersanti, A., G. Spada, R. Sabadini and M. Bonafede (1995). Global postseismic deformation, Geophys. J. Int., 120 (3), 544-566.

Piersanti, A., G. Spada and R. Sabadini (1997). Global postseismic rebound of a viscoelastic Earth: Theory for finite faults and application to the 1964 Alaska earthquake, J. Geophys. Res., 102 (B1), 477-492.

Piersanti, A. (1999). Postseismic deformation in Chile: Constraints on the asthenospheric viscosity, Geophys. Res. Lett., 26 (20), 3157-3160.

Piersanti, A., C. Nostro and F. Riguzzi (2001). Active displacement field in the Suez-Sinai area: the role of postseismic deformation, Earth and Planetary Science Letters, 193 (1-2), 13-23. 
Pollitz, F.F., R. Bürgmann and B. Romanowicz (1998). Viscosity of Oceanic Asthenosphere Inferred from Remote Triggering of Earthquakes, Science, 280 (5367), 1245-1249.

Pollitz, F.F. (2003). Transient rheology of the uppermost mantle beneath the Mojave Desert, California, Earth planet. Sci. Lett., 215 (1-2), 89-104.

Pollitz, F.F. (2005). Transient rheology of the upper mantle beneath central Alaska inferred from the crustal velocity field following the 2002 Denali earthquake, J. Geophys. Res., 110, B08407.

Pollitz, F.F., R. Bürgmann and P. Banerjee (2006). Postseismic relaxation following the great 2004 Sumatra-Andaman earthquake on a compressible self-gravitating Earth, Geophysical Journal International, 167 (1), 397-420.

Post, E.L. (1930). Generalized differentiation, Trans. Amer. Math. Soc., 32, 723-781.

Ranalli, G. (1995). Rheology of the Earth, 2nd edition, London. Riva, R.E.M. and L.L.A. Vermeersen (2002). Approximation method for high-degree harmonics in normal mode modelling, Geophysical Journal International, 151 (1), 309-313.

Soldati, G., A. Piersanti and E. Boschi (1998). Global postseismic gravity changes of a viscoelastic earth. J. Geophys. Res., 103 (B12), 29,867-29,886.

Spada, G and L. Boschi (2006). Using the Post-Widder formula to compute the Earths viscoelastic Love numbers, Geophys. J. Int., 166, 309-321.

Spada, G. (2008). ALMA, a Fortran program for computing the viscoelastic Love numbers of a spherically symmetric planet, Computers \& Geosciences, 34 (6), 667-687.

Stein, R.S. (1999). The role of stress transfer in earthquake occurrence, Nature, 402, 605-609.

Ueda, H., M. Ohtake and H. Sato (2003). Postseismic crustal deformation following the 1993 Hokkaido Nansei-oki earthquake, northern Japan: Evidence for a low-viscosity zone inthe uppermost mantle, J. Geophys. Res., 108 (B3), 2151.

Velicogna, I. and J. Wahr (2002). Postglacial rebound and Earth's viscosity structure from GRACE, J. Geophys. Res., 107 (B12), 2376.

Vergnolle, M., F. Pollitz and E. Calais (2003). Constraints on the viscosity of the continental crust and mantle from GPS measurements and postseismic deformation models in western Mongolia, J. Geophys. Res., 108 (B10), 2502.

Wahr, J. and M. Wyss (1980). Interpretation of Postseismic Deformation With a Viscoelastic Relaxation Model, J. Geophys. Res., 85 (B11), 6471-6477.

Walters, R.J., J.R. Elliott, N. D'Agostino, P.C. England, I. Hunstad, J.A. Jackson, B. Parsons, R.J. Phillips and G. Roberts (2009). The 2009 L'Aquila earthquake (central Italy): A source mechanism and implications for seismic hazard, Geophys. Res. Lett., 36, L17312.

Wessel, P. and W.H.F. Smith (1991). Free software helps map and display data, Eos, Trans. Am. Geophys. Un., 72 (41), 441. Widder, D.W. (1934). The inversion of the laplace integral and the related moment problem, Trans. Amer. Math. Soc., 36, 107-200.

Yuen, D.A. and W.R. Peltier (1982). Normal modes of the viscoelastic Earth. Geophys. J. R. astr. Soc., 69, 495-526.

Corresponding author: Dr. Valentina Cannelli, Istituto Nazionale di Geofisica e Vulcanologia, sezione di Roma, Italy; e-mail: valentina.cannelli@ingv.it

C) 2010 by the Istituto Nazionale di Geofisica e Vulcanologia. All rights reserved. 